\title{
Chile, Haití, Christchurch y Katrina: una aproximación a un análisis contrafactual
}

José Acevedo*

\section{RESUMEN}

Se presenta una propuesta de una sociedad resiliente frente a los riesgos, seguida de un resumen de la metodología supuesta por el institucionalismo histórico para el análisis de coyunturas críticas, enfatizando en el peso que tienen los desastres como productores de esas coyunturas. En su mayoría los datos han sido obtenidos del análisis de fuentes secundarias. En la descripción de cada caso se identifican las coyunturas críticas y se finaliza con el análisis contrafactual, tratando de identificar las decisiones viables que pudieron tomarse en cada una de las coyunturas y que hubieran provocado una disminución apreciable en el nivel de pérdidas ocurridas en cada uno de los cuatro casos. Finalmente las conclusiones del estudio, enfatizando la importancia del análisis contrafactual como medio para construir sociedades más resilientes.

\section{Palabras clave: Análisis contrafactual, coyunturas críticas, resiliencia}

\section{ABSTRACT}

A proposal of a resilient society against risks is presented, followed by a summary of the methodology assumed by the historical institutionalism for the analysis of critical junctures, emphasizing the weight of disasters as producers of these junctures. Most of the data have been obtained from the analysis of secondary sources. In the description of each case, critical junctures were identified, and the end result is the counterfactual analysis, trying to identify viable decisions that might have taken place in the junctures and that could have provoked a significant decrease in the level of losses in each of the four cases. Finally the study concludes emphasizing the importance of counterfactual analysis as a way to build more resilient societies.

\section{Key words: Counterfactual analysis, critical junctures, resilience}

\footnotetext{
* Universidad Nacional Autónoma de Honduras. Facultad de Ciencias Sociales, Escuela de Sociología. joalac@yahoo.com
} 


\section{INTRODUCCIÓN}

Este breve análisis cualitativo pretende responder a lo que debió hacerse 60 años atrás para sobrellevar de mejor manera cada evento (Katrina, Christchurch, Haití y Chile) tratando de ser riguroso al identificar las medidas viables que pudieron tomarse para disminuir las pérdidas humanas y de bienes, para lo que utilizaremos un modelo de sociedad resiliente, como referencia para la toma de decisiones institucionales.

\section{El modelo de la sociedad resiliente}

Una sociedad que por sus características tiene la capacidad de minimizar el impacto de cualquier evento y de recuperarse en un plazo más corto, en comparación con otras sociedades cuando enfrentan eventos naturales, posee un modelo de sociedad resiliente; este modelo puede actuar como un referente contrafactual global dado que muestra, cómo sería una sociedad que no sólo hace una gestión óptima de la reducción de riesgos de desastres, sino que ha evolucionado para minimizar los riesgos y su impacto.

La propuesta de la sociedad resiliente se apoya en la idea de la resiliencia como el resultado del modelo de desarrollo de una sociedad, presentando las siguientes las características:

1. Elevados niveles de desarrollo económico, sustentados en una población con niveles apropiados de salud y educación, capaz de realizar constantes innovaciones tecnológicas amigables con el medio ambiente.

2. Planificación del desarrollo, incorporando transversalmente la gestión de riesgos tanto a nivel nacional como local. Esta planificación incluye el territorio, con la finalidad de hacer un uso eficaz del mismo, minimizando los impactos ambientales negativos y los riesgos derivados del uso inapropiado del territorio, o bien, estableciendo las medidas apropiadas para reducir esos riesgos.

3. Bajos niveles de desigualdad, expresados en una elevada equidad en la distribución del ingreso, género, acceso a educación y salud. Estas condiciones, a su vez, se expresarán en un elevado índice de desarrollo humano (IDH) y pobreza reducida.

4. Gobernabilidad apropiada, expresada en un estado que se suma a los esfuerzos internacionales para la reducción de riesgos de desastres, es decir, que firma y respeta acuerdos internacionales relativos al desarrollo y gestión 
de riesgos. Respetando los derechos humanos, con políticas funcionales enfocadas en promover el respeto a la diversidad y, además, ejecuta políticas de protección social. De igual manera es una sociedad que hace gestión prospectiva del riesgo.

5. Superación de los procesos de toma de decisiones en compartimiento estancos, favoreciendo la coordinación transversal entre las diferentes instituciones, aumentando así, la eficacia de las acciones del estado y de la sociedad civil.

6. Preparación para una gestión prospectiva de los riesgos de desastres, mediante la integración de la gestión ambiental y la gestión de riesgos, con un enfoque armónico de reducción de riesgos de desastres para el corto y largo plazo. Por tanto, es una sociedad con conciencia del riesgo, sistemas de alerta temprana, enfocada en la resiliencia y capacidad para investigar e invertir en la reducción de los riesgos.

\section{METODOLOGÍA}

\section{La pregunta de investigación}

El presente trabajo se enfoca en responder, para cada uno de los cuatro casos de estudio, la siguiente pregunta: ¿qué debería haberse hecho en los 60 años previos a cada evento que habría disminuido las pérdidas humanas y de bienes a un $10 \%$ de las pérdidas actuales incurridas?

\section{Método para el análisis de las coyunturas críticas}

El método se basa en la propuesta del institucionalismo histórico, tal como ha sido explicado por Capoccia \& Kelemen, (2007). A continuación se presentan las ideas clave:

1. La diferencia entre coyuntura y estructura. Las sociedades siguen un proceso de desarrollo cíclico, en el cual se pueden distinguir dos fases, la del equilibrio en el cual actúan las fuerzas de las estructuras sociales, económicas, políticas y culturales. Estos procesos que responden a las estructuras son los que marcan las tendencias o también conocidos como "long periods of path-dependent institutional stability". La segunda fase, de corta duración, conocida como coyuntura crítica, relaja las tendencias estructurales, rompiendo con el equilibrio pre-existente, y permitiendo que las decisiones tomadas por actores clave con poder, frente a varias opciones, provoquen alteraciones importantes en la dirección de la vida social, aunque esto no signifique necesariamente cambio social. (Capoccia \& Kelemen, 
2007, pp. 341-343).

2. La definición de coyuntura crítica. Capoccia y Kelemen definen coyuntura crítica como: "..., períodos de tiempo relativamente cortos durante los cuales existe una probabilidad sustancialmente elevada que las elecciones de los agentes afecten el resultado de interés" (2007). A partir de esta definición, los autores proponen tener en cuenta, cuatro áreas claves para el análisis: "las asimetrías de poder, horizontes de tiempo, unidades de análisis, y los cuasi accidentes" (Capoccia \& Kelemen, 2007). Los autores hacen énfasis en la especificación de la unidad de análisis para la cual la coyuntura es crítica. Esta pueden ser desde una institución, un conjunto de instituciones, políticas públicas o un régimen político (Capoccia \& Kelemen, 2007). En cuanto al horizonte de tiempo, plantean que las coyunturas son períodos de corta duración considerados frente a los procesos estructurales, los cuales son de larga duración (Capoccia \& Kelemen, 2007). En cuanto a los cuasi-accidentes ("near misses") indican que debido a que las coyunturas son contingentes, dan lugar a múltiples posibles cursos de acción, de manera que aunque es posible que las decisiones desaten un cambio social, puede ocurrir igualmente que conduzcan a la situación anterior a la coyuntura. Las asimetrías de poder, afirman que el análisis de las coyunturas críticas debe tener en cuenta las decisiones de los actores con poder y, por tanto, las diferencias de poder entre ellos (Capoccia \& Kelemen, 2007).

\section{La reconstrucción sistemática de las decisiones durante las coyunturas} críticas. Dado que las coyunturas críticas están caracterizadas por elevados niveles de incertidumbre, el análisis de las decisiones debe basarse en la reconstrucción "..., de manera sistemática y rigurosa, cada paso del proceso de toma de decisiones, identificará, qué decisiones fueron las opciones más influyentes y cuáles estaban disponibles y viables para los actores que los tomaron, y aclarar tanto su impacto y su conexión con otras decisiones importantes.... También el análisis de contingencia: en las coyunturas críticas, las decisiones son tomadas en una situación de gran incertidumbre e imprevisibilidad, debido a la relajación de las limitaciones estructurales e institucionales "normales" de la acción "(Capoccia \& Kelemen, 2007).

4. El análisis contrafactual debe ser válido. Para que el análisis de coyunturas críticas sea válido debe existir consistencia teórica e histórica. Esto es, que las opciones para la toma de decisiones no solamente hayan existido, sino que también sean aceptables de acuerdo con la teoría (Mahoney en Capoccia y Kelemen).

5. Los desastres y las coyunturas críticas. Ese planteamiento debería ser 
complementado con un enfoque para el análisis de coyunturas críticas enfocado en la reducción de riesgos de desastres. Las catástrofes son productores de coyunturas críticas: "Así como un gran terremoto permite poner a prueba la calidad de las construcciones e infraestructura de la zona afectada de manera análoga, los desastres constituyen instancias que otorgan a las ciencias sociales la oportunidad de estudiar un sistema social o político durante periodos de crisis, revelando y poniendo a prueba también instituciones, liderazgos y procesos básicos como la reacción de las organizaciones y personas y la articulación de información" (Funk \& Figueroa, 2010, p. 79).

\section{Análisis de los casos}

\section{Chile}

1. Descripción del caso. El 27 de febrero del 2010 un terremoto de magnitud 8.8 y un tsunami afectaron a Chile, provocando 562 muertos y desaparecidos, más daños estimados en 30 mil millones de dólares. La zona de impacto humano fue la ciudad de Concepción. Vale destacar que muchas de las muertes registradas se dieron no por los efectos del terremoto mismo, sino, del maremoto que se produjo inmediatamente después. En particular, se cuestionó el papel de la Oficina Nacional de Emergencia (ONEMI) y del Servicio Hidrográfico y Oceanográfico de la Armada (SHOA), y surgieron además cuestionamientos respecto al desempeño de las autoridades militares, políticos y de seguridad (Funk \& Figueroa, 2010).

Chile es un país de alta sismicidad, puesto que experimentó 6 terremotos de gran magnitud en el período 1960-2007. Esta característica ha facilitado que Chile disponga y aplique un código de construcción con normas para resistir a los sismos. No obstante, no hay un sistema normativo coherente y tampoco articulación entre las instituciones responsables de la gestión de riesgos ((UNISDR), Secretaría de la Estrategia Internacional de las Naciones Unidas para la Reducción del Riesgo de Desastres, 2010).

2. Las coyunturas críticas. Este análisis sugiere la existencia de varias coyunturas críticas para la gestión de riesgos: La primera, generada con el terremoto de 1960, acompañado también de un tsunami. Durante esta coyuntura se creó el embrión de lo que sería más adelante la Oficina Nacional de Emergencia del Ministerio del Interior (ONEMI). La segunda, por el terremoto de 1971 y habría durado hasta 1974, cuando se produjo la creación de la ONEMI (CHILE, 2002), aunque lamentablemente con una orientación para la respuesta a emergencias ((UNISDR), Secretaría de la Estrategia Internacional de las Naciones Unidas para la Reducción del Riesgo de Desastres, 2010). Esta coyuntura se superpuso con una coyuntura de 
mayor envergadura, iniciada con el golpe de estado de septiembre de 1973, y sumado a esto los procesos de privatización en Chile, tuvieron consecuencias sobre la gestión de riesgos porque al "externalizar" varias de las actividades estatales relativas a este tema, debilitaron la capacidad de éste para realizar una acción prospectiva.

Una tercera coyuntura crítica, probablemente, se creó con el terremoto de Antofagasta de 1995, el cual tuvo una magnitud de 8.0. Esta coyuntura se habría cerrado con la emisión en 1996 de la norma NCh 433, la cual regula el diseño antisísmico de los grandes edificios.

Una cuarta coyuntura, estaría asociada a la aprobación del Plan Nacional de Protección Civil en 2002, el cual establece el diseño institucional y operativo para la gestión de riesgos en Chile, que resultó notoriamente inapropiado frente al terremoto y tsunami del 2010.

\section{Análisis contrafactual: las decisiones que hubieran cambiado el curso de la historia. En la primera coyuntura, las autoridades estatales y los empresarios de la construcción debieron lograr consenso acerca de la emisión de un sistema normativo para construcciones sismo-resistentes, para responder a futuros terremotos de gran magnitud como el de 1960 (gestión prospectiva). Este sistema normativo debió combinarse con normas enfocadas en el mejoramiento estructural de las viviendas y edificaciones existentes (gestión correctiva).}

En cuanto a la segunda coyuntura, resultado de la crisis provocada por el terremoto de 1971, se creó la ONEMI en 1974. Las autoridades estatales (ministerios, regiones y municipios) y sociedad civil pudieron decidir acerca de las capacidades y recursos de la ONEMI para una gestión eficaz de los riesgos de desastres, mediante la creación de un sistema nacional de gestión de riesgos, muy descentralizado, con un pequeño pero eficiente equipo central de coordinación dotado de poder.

A partir de la crisis provocada por el terremoto de Antofagasta de 1995, se emitieron nuevas normas antisísmicas. Esta decisión tomada mediante el acuerdo de autoridades estatales y empresarios de la construcción, debió ser de mayor alcance para obligar al mejoramiento de los diseños de las estructuras de hormigón en grandes edificaciones, reforzamiento de edificaciones de mampostería, reposición de las viviendas construidas con adobe y prohibición estricta de construcción de edificios en suelos con baja capacidad soportante. 
La coyuntura abierta con el proceso de discusión y aprobación del Plan Nacional de Protección Civil en 2002, pudo haber sido aprovechada por las autoridades estatales (ministerios, regiones y municipios) y sociedad civil para acordar un diseño simplificado de los mecanismos de coordinación institucional, la simplificación de los sistemas de alerta temprana y elaboración de un protocolo para la transmisión de información entre los organismos técnico-científicos, los organismos políticos y la población. Además, pudieron ordenar el diseño y operación de un sistema de alerta de tsunami, para las zonas turísticas con limitado acceso a comunicación electrónica.

\section{Haití}

1. Descripción del caso. El 12 de enero del 2010, Haití recibió el impacto de un terremoto de magnitud 7.0, siendo la zona de impacto humano, la capital Puerto Príncipe. La cantidad de muertos fue de más de 200,000. Haití ocupa el lugar 148 de 172 países en el Índice de Desarrollo Humano del 2010, con la mitad de su población viviendo en pobreza absoluta. El país tiene una elevada vulnerabilidad frente a huracanes, terremotos y crisis política (Banco Mundial, 2011, p. 1). La primera, determinada por la deforestación (Justice, p. 2). La población haitiana desde 1954 hasta el 2008, ha sido severamente afectada por al menos 13 huracanes y tormentas tropicales. y la segunda, debido a la falla de Enriquillo (Sociedad Dominicana de Geología (SODOGEO), 2007, p. 1). La tercera, no calificada como fenómeno natural es la inestabilidad política, marcada por una larga dictadura familiar y una crisis política permanente desde la finalización de la dictadura en 1986.

2. Las coyunturas críticas. La primera coyuntura, corresponde al período comprendido entre 1998 (huracán George), el cual causó un desastre de gran magnitud, y el 2001 cuando se estableció el Sistema Nacional de Gestión de Riesgos, este fue organizado para el manejo de riesgos de desastres y la respuesta a emergencias. Sin embargo, se caracteriza por una "... baja capacidad de respuesta y sufre de falta de apoyo institucional. Sin embargo, hay que señalar que la inestabilidad política siempre hubiera constituido un gran desafío para mantener un sistema eficiente.

La otra coyuntura relevante es la incorporación de Haití a la iniciativa HIPC en 2006, y que tuvo como resultado la elaboración de la Estrategia para la Reducción de la Pobreza, la cual, le permitió llegar al punto de culminación para obtener una condonación de alrededor de 900 millones de dólares (CEPAL, 2009). Esta 
estrategia estableció como una de sus áreas temáticas transversales: los desastres y la gestión de riesgos (Fondo Monetario Internacional, 2008). En que se establecieron los riesgos y vulnerabilidades, así como los problemas asociados al modelo de urbanización haitiano.

3.Análisis contrafactual: decisiones que hubieran cambiado el curso de la historia. Durante la primera coyuntura, la principal decisión tomada fue la creación del Sistema Nacional de Gestión de Riesgos, con la participación de 10 ministros y el presidente de la Cruz Roja de Haití. Estos actores debieron establecer a cuál ministerio le correspondería la responsabilidad del sistema, para tomar decisiones sobre mecanismos de coordinación institucional, sensibilización de la población sobre riesgos, difusión de información sobre riesgos de desastres, el desarrollo descentralizado del sistema debido a la debilidad del gobierno central y, el diseño y aplicación de normas de construcción para asegurar la resistencia a sismos. Por otra parte, también debió establecerse normas severas sobre uso del suelo a nivel urbano.

Durante la segunda coyuntura, la principal decisión fue la elaboración de la Estrategia para la Reducción de la Pobreza, la cual contiene lineamientos para la reducción de riesgos y asignación de recursos de condonación para combatir la pobreza. (Fondo Monetario Internacional, 2008). Siendo los actores con mayor poder quienes tomaron decisiones para la provisión de recursos para el desarrollo descentralizado de la gestión de riesgos, considerando las fallas de los sistemas nacionales. Por otro lado, debieron aprovechar la oportunidad para el diseño y aplicación de normas de construcción sismo-resistentes, para las viviendas construidas para la población pobre.

\section{Christchurch}

1. Descripción del caso. En septiembre del 2010, la ciudad de Christchurch, Nueva Zelanda, fue afectada por un terremoto de magnitud 7.1, sin que hubiera muertos. Nuevamente en febrero del 2011, esta ciudad fue afectada por un terremoto de magnitud 6.1 (Degenkolb, 2011, p. 1). Provocando la muerte de 181 personas. Con una significativa historia de terremotos a lo largo de los últimos 50 años; los dos más fuertes fueron en mayo de 1968, con una magnitud de 7.1 y el de julio de 2009 con una magnitud de 7.8. El primero causó pocos daños (2 muertos y 3 millones de dólares en pérdidas de ese año), el segundo, aunque fue de mayor magnitud, no causó muertos. Ambos ocurrieron en zonas despobladas, pero sí, ocasionó daños en infraestructura (Praysetya, etal.1973). 
Partiendo del planteamiento esbozado en las páginas anteriores, acerca de la asociación entre resiliencia y desarrollo, es claro que en Nueva Zelandia hay una combinación apropiada de factores de desarrollo, que la han convertido en una sociedad con un elevado nivel de resiliencia frente a los desastres. Este país expresa un elevado índice de desarrollo humano.

2. Las coyunturas críticas. La primera conyuntura, fue la iniciada con el terremoto de Inangahua de mayo de 1968 con magnitud 7.1, el cual, provocó múltiples daños en viviendas e infraestructura. Como resultado, se emitió una ordenanza que obligó al reforzamiento de los edificios de mampostería en Nueva Zelandia, los cuales tuvieron un buen desempeño durante el terremoto del 2011 en Christchurch (Degenkolb, 2011, p. 5).

Una segunda coyuntura, fue la que se inició con la crisis provocada por el terremoto de Edgecumbe de marzo de 1987, con una magnitud de 6.5. Este causó daños estructurales importantes en los pueblos de Edgecumbe, Te Teko, Kawerau, Matata y Thornton, aunque no causó ninguna muerte.

\section{Análisis contrafactual: decisiones que hubieran cambiado el curso de la} historia. En cuanto a la primera coyuntura, que se inició con el terremoto de 1968, hay varios temas en los cuales las autoridades estatales, organizaciones de sociedad civil y los empresarios, pudieron incidir en disminuir el impacto del terremoto del 2011. El primero se refiere a la existencia de normas respecto al uso del suelo, especialmente en las zonas urbanas, reduciendo la cantidad de población y estructuras expuestas a elevados niveles de riesgo. Otro tema es el mejoramiento de los códigos de construcción, con normas de diseño más exigentes para que las estructuras respondieran mejor a los requerimientos provocados por los terremotos posteriores a 1968, especialmente considerando la gran propensión del suelo a la licuefacción. Esta decisión debió acompañarse de una mayor inversión en investigación sobre el comportamiento de diversas estructuras ante diversos tipos de terremotos, de modo que las normas de diseño incluyeran decisiones para asegurar mayor sismo-resistencia de las estructuras.

Una de las decisiones positivas durante la coyuntura de 1968, fue la emisión de una ordenanza de reforzamiento de los edificios de mampostería, los que tuvieron un buen desempeño durante el terremoto del 2011 (Degenkolb, 2011, p. 5). Esta decisión debió tomarse para el reforzamiento de todos los edificios, incluyendo los de alto valor histórico.

La decisión sobre el mejoramiento de los códigos de construcción, elevando los 
requerimientos de calidad estructural y los estudios previos de suelos, pudo ser la clave para reducir el impacto del terremoto del 2011. Si antes de mediados de la década de 1980 se hubieran incorporado conceptos sísmicos modernos en los códigos de construcción, muchos edificios construidos antes de la mitad de esa década, hubieran tenido un mejor desempeño, dado que los daños en estructuras de concreto reforzado se concentraron en las edificaciones previas a esa época (Degenkolb, 2011). Hay que agregar que el colapso de dos edificios de este tipo, provocó cerca de la mitad de las muertes derivadas del terremoto.

Un tercer tema importante, es el de los daños experimentados por las líneas vitales en Christchurch, debido a la licuefacción. Dos decisiones parecen haber sido claves, si se hubieran tomado. La primera, pudo ser un desarrollo urbano más resiliente mediante una zonificación que redujera las inversiones y poblamiento en las zonas más propensas a la licuefacción y la segunda decisión, pudo ser la construcción de sistemas redundantes para las líneas vitales. Estas decisiones debieron involucrar a las autoridades de la ciudad, pero también a los empresarios y grupos de sociedad civil vinculados al desarrollo urbano.

La crisis provocada por el terremoto de 1987, inició una coyuntura interesante que estimuló la emisión de reglamentos de construcción relativos a estructuras de concreto y acero, para hacer frente a los terremotos. Desafortunadamente, estos reglamentos fueron adoptados por la mayoría de las autoridades locales, de manera que la decisión que faltó fue la adopción de tales normas como estándares nacionales (Gregory MacRae, August 2011, p. 4).

\section{Katrina}

1. Descripción del caso. En agosto del 2005, el huracán Katrina afectó los estados de Luisiana y Mississippi, provocando 1,833 muertos. La ciudad más afectada fue Nueva Orleans. Además de los muertos, hubo miles de casas destruidas y miles de heridos (Funk \& Figueroa, 2010, p. 82).

2. Las coyunturas críticas. El análisis se sustenta en una catástrofe cuya primera coyunturas se identifica como las fallas del sistema de diques y canales (Tierney, 2008, p. 121), cuya prevención y respuesta trasciende las posibilidades de los gobiernos locales y estatales, más relacionadas con decisiones que corresponden al gobierno federal, y considerando que se inició en 1965, con la aprobación de la Flood Control Act, que sirvió de fundamento para la ampliación de las obras de protección contra inundaciones, por el ejército de los Estados Unidos. Las obras realizadas en 1965, contribuyeron a una mayor expansión de la ciudad de New 
Orleans hacia la parte más baja del río Mississippi (Mlakar, 2006, p. 16). La segunda, a partir del ataque de septiembre del 2001, que incrementó la demanda de planes para el manejo de grandes eventos de riesgo. En respuesta a esta demanda, el gobierno federal elaboró un nuevo Plan de Respuesta Federal (Tierney, 2008, p. 130). Sin embargo, ese plan no fue pensado para las catástrofes provocadas por eventos naturales.

La última coyuntura, corresponde al desarrollo e impacto del huracán. El caso de Katrina es muy especial puesto que la coyuntura crítica más importante se produjo justo antes de la crisis, pero no fue reconocida como tal. Es preciso recordar que las crisis provocadas por los huracanes son de desarrollo lento, de modo que crean una coyuntura previa al impacto. Probablemente, el problema fundamental es que los actores con más poder no pudieron reconocer que estaban frente a una crisis potencial de gran magnitud (Sobel \& Leeson, 2006, p. 1).

\section{Análisis contrafactual: decisiones que hubieran cambiado el curso de la} historia. En 1965, en lugar de autorizar más y mayores obras, tal vez debió tomarse la decisión de reorientar el desarrollo de Nueva Orleans, hacia zonas más altas. Las decisiones debieron orientarse a modificar el desarrollo socioeconómico y urbano, mediante cambios en el uso del suelo y creando incentivos para la relocalización de inversiones significativas o para asegurar que las nuevas inversiones se ubicaran en zonas menos vulnerables. Estas decisiones debieron ser tomadas por las autoridades de la ciudad y el Estado, pero también por algunas agencias del gobierno federal involucradas en la planeación del desarrollo.

Para la segunda, preliminarmente, se puede identificar dos decisiones que deben haberse tomado, por lo menos desde el 9/11, debió ser la reforma de FEMA o del sistema nacional de gestión de riesgos, transfiriendo más recursos y poder de decisión a los Estados y a los gobiernos locales. Además tiene que ver con el desarrollo de planes detallados para la respuesta a catástrofes, que tuvieran en cuenta las prioridades políticas y estratégicas de los diversos actores. Estas decisiones corresponden al gobierno federal.

En el caso de la tercera coyuntura, hay varias decisiones clave que se pudieron tomar antes del 29 de agosto, que no hubieran evitado las pérdidas en infraestructura, y mucho más en vidas humanas. Identifico tres decisiones cruciales: establecer un mando único entre las organizaciones y actores involucrados en la preparación y respuesta; aceptación y movilización inmediata de la ayuda ofrecida por actores externos a la ciudad y, aplicación de un decreto de evacuación forzosa, con la movilización de los militares. 


\section{CONCLUSIONES}

1. Este análisis identifica, en dos casos de desastres y dos de catástrofes, las decisiones que pudieron tomarse en los últimos 60 años y que hubieran contribuido a disminuir el impacto de esos eventos. La metodología utilizada tiene como marco de referencia el aparato conceptual de la economía política aplicada a la gestión de riesgos. Con este marco propongo un modelo de sociedad resiliente, el cual puede orientar la toma de decisiones durante coyunturas críticas para mejorar el desempeño de las sociedades ante los riesgos.

2. El desastre en Chile, se produjo en una sociedad con un considerable nivel de resiliencia, a pesar de su modelo de desarrollo productor de vulnerabilidad. En este caso se puede afirmar que si durante las coyunturas críticas se hubieran elaborado normas de construcción con mayores niveles de seguridad, procesos de coordinación institucional más eficaces y protocolos de información y alerta temprana más funcionales, se hubieran reducido notablemente las muertes provocadas por la destrucción de edificios y por el tsunami.

3. Haití, es la antítesis de una sociedad resiliente, debido a su inestabilidad política marcada por una larga dictadura familiar y una crisis política, provocando desequilibrio o inestabilidad. En las coyunturas críticas debió enfatizarse en la aprobación de códigos de construcción para edificar viviendas e inmuebles más seguros; normas severas sobre uso del suelo a nivel urbano y por último el desarrollo de capacidades a nivel local, considerando las debilidades institucionales a nivel central.

4. En Nueva Zelandia, hay una combinación apropiada de factores de desarrollo, que la han convertido en una sociedad con un elevado nivel de resiliencia frente a los desastres. A pesar de la resiliencia resultante del elevado nivel de desarrollo, una combinación de decisiones sobre normas de construcción, enfocadas en la calidad de las estructuras y en la capacidad soportante de los suelos, más modificaciones en las normas de uso de los suelos en las zonas urbanas, hubieran provocado una disminución significativa en las pérdidas humanas y materiales.

5. A pesar de los elevados niveles de resiliencia de la sociedad estadounidense, en Nueva Orleans se produjo una catástrofe con Katrina, la cual se pudo evitar si se hubieran tomado las decisiones apropiadas durante las coyunturas críticas. Si se hubieran tomado decisiones oportunas sobre reorientación del desarrollo territorial de la ciudad, obras de ingeniería más grandes, mejor preparación para la respuesta y medidas obligatorias de evacuación de 
emergencia hacia zonas más seguras, se hubiera disminuido notablemente el alcance de las pérdidas.

6. Finalmente, hay que apuntar que dadas las limitaciones de un estudio como el presente, en el futuro debería realizarse investigaciones de mayor duración y detalle, sobre los procesos de toma de decisiones que contribuyen a la resiliencia. Es necesario avanzar en la comprensión del encadenamiento de coyunturas críticas en sociedades como la haitiana, que parece haber estado en una crisis política permanente hasta hace pocos años y, además, experimenta crisis por desastres, las cuales están igualmente encadenadas debido a la recurrencia de las tormentas tropicales y al elevado nivel de exposición de riesgo de su población.

\section{BIBLIOGRAFÍA}

Banco Mundial. (2011, noviembre). Emergency project paper.

Capoccia, G., \& Kelemen, R. D. (2007). The Study of Critical Junctures. Theory, Narrative, and Counterfactuals in Historical Institutionalism. World Politics, 59 (3), 341-369.

Castaño Guillén, Julián (2006). La dirección de resultados en las empresas privatizadas. Tesis doctoral. Universidad de Extremadura.

CEPAL. (1997). Información para la gestión del riesgo de desastres. Estudios de caso de cinco países.

CEPAL. (2009). Balance preliminar de las economías de América Latina y el Caribe 2009.

Chile, P. D. (2002). Plan nacional de proteccion civil. Santiago, Chile.

Congleton, R. D. (2006). The Story of Katrina: New Orleans and the Political Economy of Catastrophe. Public Choice, 127:5-30.

DARA. (n.d.). Earthquake in Haiti. Recuperado de

\section{http://daraint.org/2010/03/03/708/earthquake-in-haiti/}

Degenkolb. (2011). Christchurch earthquake. Perspectives, 9.

Deren, Jane. Hurricanes and Haiti: A Tragic History. Solidarity , 1-2. Recuperado de http://www.loyola.edu/ccsj/HaitiRelief/HaitiHurricanes08_0.pdf

Fondo Monetario Internacional. (2008). Haiti: Poverty Reduction Strategy Paper. IMF Country Report. Fordyce, E., Sadiq, A.-A., \& Chikoto, G. Haiti's Emergency Management: A Case of Regional Support, Challenges, Opportunities, and Recommendations for the Future.

Funk, R. L., \& Figueroa, P. (2010). Coyunturas críticas de un desastre: El caso del 27F. Revista Chilena deAdministración Pública, pp. 69/93.

Geonet. M 6.5, Edgecumbe, 2 March 1987. Recuperado de http://info.geonet.org.nz/display/quake/M+6.5\%2C+Edgecumbe $\% 2 \mathrm{C}$ 
+2+March+1987. (n.d.)

Geonet. M 6.7, Gisborne, 20 December 2007. Recuperado de

http://info.geonet.org.nz/display/quake/M+6.7,+Gisborne,+20+Dece mber+2007. (n.d.)

Geonet. M 7.1, Inangahua, 24 May 1968. Recuperado de

http://info.geonet.org.nz/display/quake/M+7.1\%2C+Inangahua $\% 2 \mathrm{C}+$ 24+May+1968.

Gregory MacRae, C. C. (August 2011). Review of NZ Building Codes of Practice.

Report to the Royal Commission of Inquiry into the Building Failure Caused by the Christchurch Earthquakes

Mlakar, P. F. (2006). The Behavior of Hurricane Protection Infrastructure in New Orleans. The Bridge, $36(1), 14-20$.

NCDC. Landfalling Puerto Rico / Dominican Republic / Haiti Hurricanes (Since 1950). Recuperado de

http://www.ncdc.noaa.gov/oa/satellite/satelliteseye/educational/ landPRHIS.html

OPS. (2010). El terremoto y tsunami del 27 de febrero en Chile. Crónica y lecciones aprendidas en el sector salud. OPS, Santiago.

Praysetya, G., Beavan, J., Xiaoming, W., Reyners, M., Power, W., Wilson, K., et al. (168 (2011), 1973-1987). Evaluation of the 15 July 2009 Fiordland, New Zealand Tsunami in the Source Region. Pure and Applied Geophysics.

Quigley, \& L. A. Rosenthal, Risking hose and home: Disasters, Citie. Public Policy (pp. 119-138).

Rencoret, N., Stoddard, A., Haver, K., Taylor, G., \& Harvey, P. (2010). Haiti Earthquake Response. ContextAnalysis. ALNAP y UNEG.

Sobel, R. S., \& Leeson, P. T. (2006). Government's response to Hurricane Katrina: A public choice Analysis. Public Choice , 55-73.

Sociedad Dominicana de Geología (SODOGEO). (2007). Geonoticias, 32.

Tierney, K. (2008). Hurricane Katrina: Catastrophic Impacts and Alarming Lessons. In J.M.

UNDP. New Zealand. HDI values and rank changes in the 2013 Human Development Report. Recuperado de

http://hdrstats.undp.org/images/explanations/NZL.pdf.

UNISDR, (2010). Diagnóstico sobre la situación de la reducción de riesgos de desastres.

USGS (2013). Historic Earthquakes. Recuperado de http://earthquake.usgs.gov/ earthquakes/world/events/1968_05_23.php. (n.d.) 